\title{
RIEMANN-ROCH THEOREMS FOR DIFFERENTIABLE MANIFOLDS
}

\author{
BY M. F. ATIYAH AND F. HIRZEBRUCH
}

Communicated by Hans Samelson, May 11, 1959

1. Introduction. The Riemann-Roch Theorem for an algebraic variety $Y$ (see [7]) led to certain divisibility conditions for the Chern classes of $Y$. It was natural to ask whether these conditions held more generally for any compact almost complex manifold. This question, and various generalizations of it, were raised in [8] and most of these have since been answered in the affirmative in [2] and [11].

More recently Grothendieck [3] has obtained a more general Riemann-Roch Theorem for a map $f: Y \rightarrow X$ of algebraic varieties. This reduces to the previous Riemann-Roch Theorem on taking $X$ to be a point. Grothendieck's Theorem implies many conditions on characteristic classes, and again it is natural to ask if these conditions hold more generally for almost complex or even differentiable manifolds. The purpose of this note is to enunciate certain differentiable analogues of Grothendieck's Theorem. These "differentiable Riemann-Roch Theorems" yield, as special cases, the divisibility conditions mentioned above and also certain new homotopy invariance properties of Pontrjagin classes. As an application of the latter we get a new proof (and slight improvement) of the result of KervaireMilnor [10] on the stable $J$-homomorphism.

Another differentiable Riemann-Roch Theorem, with applications to embeddability problems of differentiable manifolds, will be found in $[1]$.

The proofs of our theorems rely heavily on the Bott periodicity of the classical groups $[4 ; 5 ; 6]$, and are altogether different from the earlier methods of [2] and [11], which were based on Thom's cobordisme theory and Adams' spectral sequence.

2. Definitions. The spaces $X, Y$ considered will be countable finitedimensional CW-complexes, and for simplicity we will suppose them connected.

Following Grothendieck [3] we define an abelian group $K(X)$ as follows. Let $F(X)$ be the free abelian group generated by the set of all isomorphism classes of complex vector bundles over $X$. To every triple $t=\left(\xi, \xi^{\prime}, \xi^{\prime \prime}\right)$ of vector bundles with $\xi \cong \xi^{\prime} \oplus \xi^{\prime \prime}$ we assign the element $[t]=[\xi]-\left[\xi^{\prime}\right]-\left[\xi^{\prime \prime}\right]$ of $F(X)$, where $[\xi]$ denotes the isomor- 
phism class of $\xi$. The group $K(X)$ is defined as the quotient of $F(X)$ by the subgroup generated by all the elements of the form $[t]$; it has an augmentation $\epsilon: K(X) \rightarrow \boldsymbol{Z}$ obtained by assigning to each vector bundle the dimension of its fibre. It is then not difficult to show that the kernel of $\epsilon$ may be identified with the group of "stable" vector bundles, i.e. with the homotopy classes of maps $X \rightarrow B_{U}$, where $B_{U}$ is the classifying space of the "infinite" unitary group $U$ (see [4]).

The tensor product of vector bundles, being distributive with respect to direct sums, defines a commutative ring structure in $K(X)$; the unit 1 is given by the trivial bundle of dimension one.

If $Y$ is a (nonempty) subcomplex of $X$ we define the relative group $K(X, Y)$ to be the subgroup of $K(X / Y)$ of augmentation zero, where $X / Y$ is the space obtained from $X$ by identifying $Y$ to a point. Then, as for cohomology, one can show that $K(X, Y)$ is a $K(X)$-module.

In a similar way, using real or quaternionic vector bundles we define groups $K O(X)$ or $K S P(X)$ respectively; $K O(X)$ is also a ring (but not $K S P(X)$ ). The inclusions $i: O \rightarrow U, j: S P \rightarrow U$ induce homomorphisms $i_{*}: K O(X) \rightarrow K(X)$ and $j_{*}: K S P(X) \rightarrow K(X)$.

The Chern character (see [2]) defines a ring homomorphism ch: $K(X) \rightarrow H^{*}(X, Q)$ (rational cohomology) which commutes with augmentation; its image will be denoted by $\operatorname{ch}(X)$. The images of ch $\circ i_{*}$ and $\operatorname{ch} \circ j_{*}$ will be denoted by $\operatorname{ch} O(X)$ and $\operatorname{ch} S P(X)$ respectively.

Let $S^{2}$ denote the 2 -sphere. Then the Bott periodicity for $B_{U}$ (see $[4 ; 5 ; 6])$ may be reinterpreted to give ${ }^{1}$

Proposition 1. We have a commutative diagram:

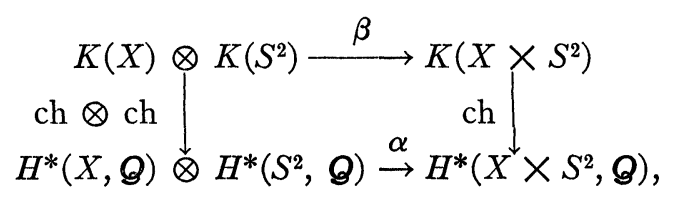

where $\beta$ is induced by the tensor product of bundles, $\alpha$ by the cup product, and both are isomorphisms.

By iteration the same result holds with $S^{2}$ replaced by $S^{2 n}$.

From now on $X, Y$ will denote compact connected oriented differentiable manifolds.

According to $[2, \S 26.5]$, an $S O(n)$-bundle is the image of a $\operatorname{Spin}(n)$ bundle if and only if the second Stiefel-Whitney class $w_{2}$ vanishes. This motivates the following definition: a continuous map $f: Y \rightarrow X$

${ }^{1}$ There are similar results for $O$ and $S P$ [see 6]. 
is called a Spin-map if $w_{2}(Y)=f^{*} w_{2}(X) . Y$ will be called a Spinmanifold if the map $Y \rightarrow$ point is a Spin-map, i.e. if $w_{2}(Y)=0$. More generally $f: Y \rightarrow X$ will be called a $c_{1}-m a p$ if we are given an element $c_{1} \in H^{2}(Y, \boldsymbol{Z})$ such that $c_{1} \equiv w_{2}(Y)-f^{*} w_{2}(X) \bmod 2 . Y$ will be called a $c_{1}$-manifold if $Y \rightarrow$ point is a $c_{1}$-map. Thus a Spin-map (or manifold) is a $c_{1}$-map (or manifold) with $c_{1}=0$.

As in [2], if $\xi$ is a real vector bundle we define

$$
\hat{\mathfrak{A}}(\xi)=\prod_{i} \frac{x_{i} / 2}{\sinh x_{i} / 2},
$$

where the Pontrjagin classes of $\xi$ are the elementary symmetric functions in the $x_{i}^{2}$. If $\xi$ is the tangent bundle of $Y$ we write $\hat{\mathfrak{A}}(Y)$ instead of $\hat{\mathfrak{A}}(\xi)$. For any $d \in H^{2}(Y, Q)$ and any $y \in \operatorname{ch}(Y)$ we define $\hat{A}(Y, d, y)$ to be the rational number obtained by evaluating the top-dimensional component of $y \cdot e^{d} \cdot \hat{\mathfrak{A}}(Y)$ on the fundamental cycle of $Y$. If $d=0, y=1$, we put $\hat{A}(Y, 0,1)=\hat{A}(Y)$.

\section{3 . Statement of results.}

TheOREM 1. Let $\operatorname{dim} Y \equiv \operatorname{dim} X \bmod 2$. Then a $c_{1}$-map $f: Y \rightarrow X$ induces a (group) homomorphism $f_{!}: \operatorname{ch}(Y) \rightarrow \operatorname{ch}(X)$ given by

$$
f:(y) \cdot \hat{\mathfrak{A}}(X)=f_{*}\left(y \cdot e^{c_{1} / 2} \cdot \hat{\mathfrak{A}}(Y)\right), \quad y \in \operatorname{ch}(Y),
$$

where $f_{*}$ is the Gysin homomorphism (the Poincare dual of the homology homomorphism).

Remarks. (1) It is probable that $f_{1}$ is actually induced by a functorial homomorphism $K(Y) \rightarrow K(X)$.

(2) If $Y$ and $X$ are complex manifolds and $f$ is a holomorphic map, then $f$ is a $c_{1}$-map in a natural way: $c_{1}=c_{1}(Y)-f^{*} c_{1}(X)$. Theorem 1 can then be rewritten as

$$
f_{1}(y) \cdot \mathfrak{T}(X)=f_{*}(y \cdot \mathfrak{I}(Y)),
$$

where $\mathfrak{T}$ is the (total) Todd class (see [2]). This is the Grothendieck formula (see [3]). However, in the Grothendieck theory (for algebraic varieties) $f_{\mathfrak{l}}$ has a direct definition in terms of coherent algebraic sheaves. This has no counterpart in the differentiable theory.

Theorem 2. (i) Let $\operatorname{dim} Y \equiv \operatorname{dim} X \bmod 2$. Then a Spin-map $f: Y \rightarrow X$ induces a (group) homomorphism $f_{1}: \operatorname{ch}(Y) \rightarrow \operatorname{ch}(X)$ given by

$$
f:(y) \cdot \hat{\mathfrak{U}}(X)=f_{*}(y \cdot \hat{\mathfrak{A}}(Y)) \text {. }
$$

(ii) If moreover 
(a)

$$
\operatorname{dim} Y-\operatorname{dim} X \equiv 0 \bmod 8,
$$

then $f_{1} \operatorname{ch} O(Y) \subset \operatorname{ch} O(X)$, while if

$$
\operatorname{dim} Y-\operatorname{dim} X \equiv 4 \bmod 8,
$$

then $f_{1} \operatorname{ch} O(Y) \subset \operatorname{ch} S P(X)$.

REMARK. Theorem 2(i) is a special case of Theorem 1, but (ii) is a further refinement.

CoRollary 1. Let $Y$ be a $c_{1}$-manifold with $\operatorname{dim} Y \equiv 0 \bmod 2$, and let $y \in \operatorname{ch}(Y)$. Then $\hat{A}\left(Y, c_{1} / 2, y\right)$ is an integer.

This follows at once from Theorem 1 by taking $X$ to be a point.

Corollary 2. (i) Let $Y$ be a Spin-manifold with $\operatorname{dim} Y \equiv 0 \bmod 2$, and let $y \in \operatorname{ch}(Y)$. Then $\hat{A}(Y, 0, y)$ is an integer; in particular $\hat{A}(Y)$ is an integer.

(ii) If moreover $\operatorname{dim} Y \equiv 4 \bmod 8$ and $y \in \operatorname{ch} O(Y)$, then $\hat{A}(Y, 0, y)$ is an even integer; in particular $\hat{A}(Y)$ is an even integer.

(i) is a special case of Corollary 1. For (ii) we use Theorem 2(ii)b, with $X=$ point; we need only observe that $\operatorname{ch} S P$ (point) $=2 Z$ (since the inclusion $S p(n) \rightarrow U(2 n)$ doubles the fibre dimension).

REMARKs. Corollary 1 was proved by Borel-Hirzebruch [2, Part II], except for the prime 2. A complete proof, including the prime 2, follows by the methods of [2] from results of Milnor [11] (see [2, Part III]). Corollary 2(ii) is new. It was conjectured in [2, Part II], where it was pointed out that it provides a satisfactory generalization of Rohlin's Theorem [12]: if $\operatorname{dim} Y=4$ and $w_{2}(Y)=0$, then $p_{1}(Y)$ $\equiv 0 \bmod 48$. Kervaire and Milnor have used Corollary 2(i) for their result on the $J$-homomorphism [10]; their result can now be sharpened by using Corollary 2(ii) (see also Corollary 5 below).

Corollary 3. Let $f: Y \rightarrow X$ be a homotopy equivalence, and identify $H^{*}(Y, \boldsymbol{Q})$ with $H^{*}(X, \boldsymbol{Q})$ under $f^{*}\left(\right.$ or $\left.f_{*}\right)$. Then $\hat{\mathfrak{A}}(Y) / \hat{\mathfrak{A}}(X) \in \operatorname{ch} O(X)$.

This follows from Theorem 2(ii)a. We need only remark that, $w_{2}$ being a homotopy type invariant [13], $f$ is a Spin-map. Corollary 3 contains new information on the Pontrjagin classes, for example:

Corollary 4. (i) The first Pontrjagin class $p_{1}$ of a compact oriented differentiable manifold $X$ (regarded as an element of $H^{4}(X, Z)$ modulo its torsion subgroup) is a homotopy type invariant mod 24.

(ii) If moreover $H^{2}\left(X, Z_{2}\right)=0$, then $p_{1}$ is invariant $\bmod 48$. 
Proof. Equating 4-dimensional components in Corollary 3 we get $p_{1}(Y)-p_{1}(X)=24 p_{1}(\xi)$ for some orthogonal bundle $\xi$. This proves (i). Also $p_{1}(\xi) \equiv w_{2}^{2}(\xi) \bmod 2$, see $[2, \S 30]$; thus $p_{1}(\xi)$ is even if $H^{2}\left(X, \boldsymbol{Z}_{2}\right)$ $=0$. This proves (ii).

REMARK. Wu [14] has proved that the $p_{i}$ are homotopy invariants mod 12. Thus, for $p_{1}$, Corollary 4 is an improvement on Wu's results. Moreover the example of $S^{4}$ bundles over $S^{4}$ shows that Corollary $4(\mathrm{ii})$ is best possible.

COROLlary 5 (KeRvaIRE-Milnor [10]). The order of the image of the stable homomorphism $J: \pi_{4 k-1}(S O(q+1)) \rightarrow \pi_{4 k+q}\left(S^{q+1}\right)(q \geqq 4 k)$ is divisible by the denominator of $B_{k} / 4 k$, where $B_{k}$ is the kth Bernoulli number.

This follows easily from Corollary 3 and the results of James and Whitehead [9] on stable sphere bundles over spheres.

4. Outline of proof. We sketch the proof of Theorem 2(i). The other proofs are similar. Since only the homotopy class of $f$ is relevant to the theorem we may suppose that $f$ is differentiable. Let $g: Y \rightarrow S^{2 n}$ be a differentiable embedding of $Y$. Then $f: Y \rightarrow X$ can be factored into the differentiable embedding $F=f \times g: Y \rightarrow X \times S^{2 n}$ followed by the projection $\pi: X \times S^{2 n} \rightarrow X$. Since Theorem 2(i) is transitive it is sufficient to prove it for the case of an embedding and for the projection $\pi$ separately. For $\pi$ the theorem is an elementary consequence of Proposition 1 (with $S^{2 n}$ replacing $S^{2}$ ), i.e. it follows from the Bott theory.

For an embedding $f: Y \rightarrow X$ Theorem 2(i) states that

$$
f_{*}\left(\operatorname{ch}(Y) \cdot \hat{\mathfrak{A}}(\xi)^{-1}\right) \subset \operatorname{ch}(X)
$$

where $\xi$ is the normal bundle of $Y$ in $X$ (and $w_{2}(\xi)=0$ since $f$ is a Spin-map). Let $A$ be a closed tubular neighborhood of $Y$ in $X$ whose boundary $E$ is the sphere bundle associated to $\xi$. Because of the natural homomorphism $\operatorname{ch}(A, E) \rightarrow \operatorname{ch}(X)$ it is sufficient to show that

$$
\phi_{*}\left(\operatorname{ch}(Y) \cdot \mathfrak{A}(\xi)^{-1}\right) \subset \operatorname{ch}(A, E),
$$

where $\phi_{*}: H^{*}(Y, Q) \rightarrow H^{*}(A, E ; Q)$ is the Thom-Gysin isomorphism [13].

Since $K(A, E)$ is a $K(A)$-module, and so a $K(Y)$-module (since $Y$ is a deformation retract of $A$ ) it is sufficient finally to construct an element $\eta \in K(A, E)$ such that

$$
\operatorname{ch} \eta=\phi_{*} \hat{\mathfrak{U}}(\xi)^{-1} .
$$


This is done by a universal construction on the classifying space of $\operatorname{Spin}(2 n)$, using the difference between the two spinor representations $\Delta^{+}$and $\Delta^{-}$of $\operatorname{Spin}(2 n)$. The formula for $\operatorname{ch} \eta$ is a consequence of the character formula:

$$
\operatorname{ch} \Delta^{+}-\operatorname{ch} \Delta^{-}=\prod_{i=1}^{n}\left(e^{x_{i} / 2}-e^{-x_{i} / 2}\right),
$$

where we adopt the notation of [2].

\section{REFERENCES}

1. M. F. Atiyah and F. Hirzebruch, Some non-embeddability theorems for differentiable manifolds, Colloque de Topologie, Lille, 1959.

2. A. Borel and F. Hirzebruch, Characteristic classes and homogeneous spaces I, Amer. J. Math. vol. 80 (1958) pp. 458-538; II and III to appear.

3. A. Borel and J-P. Serre, Le théorème de Riemann-Roch (d'après Grothendieck). Bull. Soc. Math. France vol. 86 (1958), pp. 97-136.

4. R. Bott, The stable homotopy of the classical groups, Proc. Nat. Acad. Sci. U.S.A. vol. 43 (1957) pp. 933-935. 61.

5. - The space of loops on a Lie group, Mich. Math. J. vol. 5 (1958) pp. 351959.

6. - Some remarks on the periodicity theorems, Colloque de Topologie, Lille,

7. F. Hirzebruch, Neue topologische Methoden in der algebraischen Geometrie, Springer, 1956.

8. - Some problems on differentiable and complex manifolds, Ann. of Math. vol. 60 (1954) pp. 213-236.

9. I. M. James and J. H. C. Whitehead, The homotopy theory of sphere bundles over spheres, Proc. London Math. Soc. vol. 4 (1954) pp. 196-218.

10. M. A. Kervaire and J. Milnor, Bernoulli numbers, homotopy groups and a theorem of Rohlin. Proceedings of the International Congress of Mathematicians, 1958.

11. J. Milnor, On the cobordisme ring $\Omega^{*}$, and a complex analogue, (in preparation).

12. V. A. Rohlin, Nere results in the theory of 4-dimensional manifolds, Dokl. Acad. Nauk. S.S.S.R. vol. 84 (1952) pp. 221-224 (in Russian).

13. R. Thom, Espacés fibrès en sphères et carrés de Steenrod, Ann. Sci. Ecole Norm. Sup. vol. 69 (1952) pp. 109-182.

14. W. T. Wu, On the Pontrjagin classes III, Acta Math. Sinica vol. 4 (1954) (in Chinese).

Pembroke College, Cambridge and

UNIVERSITY OF BONN 